\title{
GALÁXIAS DE HAROLDO DE CAMPOS: LA LECTURA COMO MANTRA ${ }^{1}$
}

\author{
Haroldo de Campos' Galáxias: Reading as an mantra
}

Felipe Cussen*

\section{RESUMEN}

En este ensayo analizo la lectura oral de dos fragmentos de Galáxias de Haroldo de Campos, quien las leyó acompañado por Alberto Marsicano tocando el sitar. A partir de la vinculación que el propio autor establece con los conceptos de raga y mantra, intentaré determinar hasta qué punto esta versión confirma o desmiente dicha comparación.

Palabras clave: Haroldo de Campos, Galáxias, oralización, raga, mantra.

\begin{abstract}
In this essay I analize the oral reading of two fragments of Haroldo de Campos' Galaxias, who read them accompanied by Alberto Marsicano playing the sitar. From the link that the author establishes with the concepts of raga and mantra, I will try to determine how far this version confirms or denies the comparison.

Keywords: Haroldo de Campos, Galáxias, oralization, raga, mantra.

\footnotetext{
${ }^{1}$ Este ensayo forma parte de mi proyecto "Mística, poesía y música", incluido en el proyecto Inserción de Capital Humano Avanzado en la Academia: "Fortalecimiento de las Humanidades en el Instituto de Estudios Avanzados y el Doctorado en Estudios Americanos de la Universidad de Santiago de Chile (CSA-USACH) (79100004)", financiado por Conicyt. Una versión preliminar fue leída en las VI Jornadas Brasileñas de la Universidad de Chile, el 30 de agosto de 2011. También me he referido a este tema en relación a la obra de algunos poetas contemporáneos en el ensayo "Mantras y poesía sonora" (ver referencias).

* Instituto de Estudios Avanzados, Universidad de Santiago de Chile. Santiago, Chile. Correo electrónico: felipecussen@gmail.com
}

Artículo recibido el 5 de julio de 2013. Aceptado el 28 de agosto de 2014. 
El 18 de noviembre de 1963, Haroldo de Campos escribió el primer fragmento de Galáxias. Entre noviembre de 1975 y marzo de 1976 escribió el último. Luego de algunas publicaciones parciales en la revista Invenção (en 1964 y 1966) y en sus libros Xadrez de estrelas (en 1976) y Signantia: quasi coelum (en 1979), así como de traducciones de fragmentos al alemán, francés, español e inglés, publicó la primera edición completa en 1984. En 1992 publicó el CD Isto não é um livro de viagem, que incluye su propia "oralizaçáo" de 16 fragmentos. Este CD fue incluido en la segunda edición de Galáxias el 2004, un año después de la muerte de su autor.

Durante todo este tiempo, Galáxias ha recibido una innumerable cantidad de lecturas críticas, lo que hace muy difícil encontrar alguna veta interpretativa para quienes hemos llegado tarde a su encuentro. La diversidad de interpretaciones que ha suscitado es tal que, si alguien no hubiera leído el libro pero leyera todos los comentarios que se han escrito sobre él, pensaría que se trata de libros diferentes. Si uno lo ha leído, concordará con todos, porque el texto efectivamente soporta esas múltiples lecturas.

Gran parte del interés proviene de la evolución que este libro marca en la trayectoria de su autor ${ }^{2}$. En la segunda mitad de los ańos 50 culminaba la etapa "heroica" del grupo Noigandres, fundado por Haroldo, su hermano Augusto y Décio Pignatari. En este período ellos se hicieron conocidos principalmente por la publicación de poemas visuales compuestos por muy pocos elementos verbales, expandidos simétricamente a través de grandes espacios en blanco. En Galáxias, en cambio, Haroldo retornaba al verso, mediante textos escritos en una prosa muy tupida, sin puntuación, que colmaban apretadamente cada página.

Desde entonces, esta obra ha sido comparada con el Finnegan's Wake de Joyce (Roberto Echavarren (en su prólogo a la traducción española de Galáxias), y también Marjorie Perloff), los Cantos de Pound (Fernando Pérez) y el "Coup de dés" de Mallarmé (Wladimir Krysinski, en Jackson (ed.)) hasta La Divina Comedia (Kenneth David Jackson, en Motta (ed.)), La Odisea (Luiz Costa Lima, en Jackson (ed.)) y el Génesis (Gonzalo Aguilar en Poesía concreta brasileña...). Se ha discutido su estatus ambivalente de prosa o poesía (Andrés Sánchez Robayna en su apéndice de Signantia quasi coelum (Campos), Paulo Leminski, Eduardo Milán en "Escritura, poesía, luz negra” y "El Odiseo brasileño...", al igual que Aguilar y Perloff), su condición de libro de viaje (Jacobo Sefamí), así como su apertura a la contingencia política (Aguilar, en Block de Behar (ed.)), la inclusión de la oralidad (Néstor Perlongher y Roberto Appratto), los vínculos con la cultura popular y el movimiento Tropicália (Lidia Santos) y con el neobarroco (algunos fragmentos fueron incluidos en la antología Medusario (Echavarren y otros)).

\footnotetext{
${ }^{2}$ Para una caracterización de las diversas etapas de su trayectoria, ver "Haroldo de Campos and the Poetics of Invention”, de Kenneth David Jackson (en Jackson (ed.), pp. 17-37).
} 
Varias de estas rutas de lectura surgen de las referencias intertextuales y los numerosos momentos autorreflexivos del poema, y muchas también habían sido apuntadas por el mismo Haroldo de Campos en textos críticos, notas y entrevistas, en los que no escatima los calificativos que aluden a su condición caleidoscópica: "texto em mosaico ou constelar... Periplo e palimpsesto" ("Livro de Ensaios: Galáxias", p. 41), "uma plurinarrativa e o 'grau zero' do narrar" (Metalinguagem..., p. 271), "texto imaginado no extremar dos limites da poesia e da prosa, pulsão bioescritural em expansão galática... (tendo por ímã temático a viagem como livro ou o livro como viagem, e por isso mesmo entendido também como um 'livro de ensaios'), ... uma insinuação épica que se resolveu numa epifânica” (Nota a la primera edición, incluida también en la segunda (p. 113)). El proyecto inicial consideraba que las páginas se ofrecieran sueltas, de modo que el lector pudiera leerlas en distintos órdenes, como si en cada una de ellas estuviera "contenido el libro completo y un libro diferente cada vez", y así el lector "estuviera hojeando el universo entero a partir de un punto privilegiado, con la mirada aléfica de un Borges" ("De la poesía concreta...”, p. 24). Si bien el libro se editó con sus hojas bien sujetas, éstas se mantuvieron sin numeración, con el reverso en blanco, como si pudieran arrancarse para funcionar autónomamente, aunque el texto inicial y el final, que tematizan su condición de comienzo y conclusión (y que son llamados formantes), aparecieron escritos en cursiva remarcando su inamovible posición.

Junto con las traducciones, que continuaron expandiéndose con la misma amplitud que las recepciones críticas desde diversas latitudes, fueron apareciendo algunas musicalizaciones de sus fragmentos, a cargo de Conrado Silva, Theophil Maier, Péricles Cavalcanti, Edvaldo Santana, y Caetano Veloso, con la conocida "Circuladô". Asimismo, Livio Tragtenberg creó el film musical "Um sarampo de estrelas para “Galáxias”” y Julio Bressane dirigió los vídeos Galáxia Albina e Infernalágio: logodédalo - Galáxia Dark. Aunque no he podido encontrarlos, sí es posible ver en Youtube ${ }^{3}$ una curiosa escena de otra película de Bressane, Sermóes, en las que aparece el mismísimo Haroldo de Campos con un traje y en un escenario del siglo XVII, leyendo parte del fragmento final de Galáxias. Lo que resulta más llamativo es que sentado atrás suyo se encuentra el músico Alberto Marsicano, vestido a la usanza india y tocando el sitar. Esos escasos minutos nos entregan una perfecta imagen de la sorprendente superposición de tiempos y culturas que se busca en este libro.

Este mismo dúo ${ }^{4}$ se repite el plato un par de años después en la grabación del CD Isto não é um livro de viagem, específicamente en los tracks correspondientes al

\footnotetext{
${ }^{3}$ Disponible en: http://www.youtube.com/watch?v=2fdWMq6GsdM.

${ }^{4}$ Haroldo de Campos escribió los prefacios a algunos libros de poesía (Idiomalabarismos (1979)) y traducción (Haikai (1988) y Sijô Poesiacanto coreana clásica (1994)) de Marsicano, y también le dedica un poema en el libro Crisantempo, "pré-haicai (para Marsicano)", (p. 103).
} 
formante inicial y al final. El CD incluye una interesante nota (reproducida también en la segunda edición de Galáxias) en la que Haroldo comenta en detalle los 16 fragmentos escogidos, y no trepida en calificar a su obra como "Audiovideotexto, videotextogame". Además indica que la "oralização" había estado siempre implícita en el proyecto y asegura que se trata de "um livro para ser lido em voz alta, que propóe um ritmo e uma prosódia, cujas zonas 'obscuras' se transparentam à leitura e cujas palavras, oralizadas, podem ganhar força talismânica, aliciar e seduzir como mantras" (Galáxias, p. 119). Ése es el motivo por el que convida a Marsicano, y ańade que "a mobilidade das ragas indianas, onde o aleatório é controlado por estruturas de repetição, soa congenial ao meu texto-partitura”. Finalmente, también se refiere a las numerosas palabras o frases en otros idiomas, cuyo valor es siempre "mântrico, 'transmental', ainda quando não imediatamente alcançável no nivel semântico", y que fluyen y confluyen en el ritmo general del texto (p. 119).

Son estas dos versiones sonoras y su correspondiente explicación las que me motivan a internarme un poco más en estas Galáxias. Por una parte, me llama la atención la casi nula preocupación (en comparación con las numerosísimas interpretaciones del texto escrito) que han provocado a sus comentaristas, pero además me interesan porque permiten establecer un vínculo cultural bastante específico con elementos de la cultura india (los mantras y los raga), que curiosamente ocupa un lugar muy pequeño dentro de su enorme mapa de referencias. La voracidad antropofágica de Haroldo de Campos le permitió desarrollar su actividad crítica y transcreadora en ámbitos lingüísticos como el inglés, el ruso, el francés, el provenzal, el italiano, el alemán, el español, el griego, el latín, el hebreo, e incluso acercarse a la poesía náhuatl y egipcia. Uno de sus focos más importantes en estas labores fue la cultura oriental, específicamente la poesía japonesa y aún más la china, pues recogió las ideas de Fenollosa y Pound en torno a la escritura ideogramática. Tanto en artículos dedicados al orientalismo de Haroldo (por ejemplo "Do ideograma ao fonograma: a travessia haroldiana Oriente-Ocidente", de Yun Jung Im (en Dick (ed.), pp. 242-62)), como en una entrevista suya sobre este tema con María Ester Maciel, prácticamente no existen referencias a la India, y en otra con Rodolfo Mata apenas indica de paso que tiene mucho interés "por la filosofía oriental, tanto budista como china o hindú, y por el budismo-zen en su formulación japonesa” (De la razón antropofágica, p. 212). Y dentro de sus poemas plagados de referencias culturales, apenas he encontrado "poemandala", de la serie "lacunae", que, a pesar de su título, tiene mucho más que ver con los hexagramas del I Ching que con el hinduismo (Xadrez de Estrelas, s/n).

Resulta atractivo, por otra parte, elucubrar hasta qué punto un poeta que se muestra muy alejado de una concepción metafísica o mística de la poesía pudiera tener algo en común con la cosmovisión hindú, en la que la palabra y el sonido son portadores de una carga espiritual muy fuerte. Considero valioso, además, 
intentar una interpretación que no tenga materiales suficientes para seguir al pie de la letra la autoexplicación tan rotunda y autosuficiente que Haroldo de Campos intenta establecer sobre su obra. Esta se presenta como un sistema perfectamente controlado de creación, traducción y reflexión, que reitera insistentemente sus ideas matrices para forzar una tarea crítica que se limite a la paráfrasis de sus ingeniosos juegos de palabra (cuando no provoca, por el contrario, un rechazo en bloque). En la lógica de su "plan de dominación mundial", pareciera a primera vista que su mención a los mantras y ragas fuera simplemente un gesto que buscaba colonizar una zona aún no tocada. Pero además hay un detalle interesante: hay una mención bastante anterior a los mantras en un texto crítico de Severo Sarduy, publicado como apéndice en Signantia: quasi coelum, en la que califica a Galáxias como "mar... em altazor: palavras engastadas como mantras ou talismás fônicos, marulhar de transmutaçóes" (p. 124). Este comentario proveniente de un escritor que sí tuvo un interés profundo en la India (al igual que Octavio Paz, otro amigo de Haroldo), sumado al acompańamiento de Marsicano, pueden hacernos pensar que, más que asumir de manera profunda la posibilidad de que su lectura comparta los presupuestos del hinduismo, se trata de una impresión más apurada y superficial, que no pudo dejar de anotar quizás como una simple intuición.

Para avanzar en esta reflexión, citaré el inicio de Galáxias:

e começo aqui e meço aqui este começo e recomeço e remeço e arremesso

e aqui me meço quando se vive sob a espécie da viagem o que importa

náo é a viagem mas o começo da por isso meço por isso começo escrever

mil páginas escrever milumapáginas para acabar com a escritura para

começar com a escritura para acabarcomeçar com a escritura por isso

recomeço por isso arremeço por isso teço escrever sobre escrever é

o futuro do escrever sobrescrevo sobrescravo em milumanoites miluma-

páginas ou uma página em uma noite que é o mesmo noites e páginas

mesmam ensimesmam onde o fim é o comêço onde escrever sobre o escrever

é não escrever sobre náo escrever e por isso começo...

(p. 13).

Si uno lee en silencio este texto repetitivo y aliterativo, lo más probable es que tenderíamos a marcar un ritmo apurado y ansioso. La lectura de Haroldo5, "comentada" por los acordes de Marsicano, se presenta mucho más lenta y ceremoniosa, acentuando la condición de apertura que ocupa esta parte

\footnotetext{
${ }^{5}$ El CD está disponible íntegramente en la página UBUweb; los tracks de los dos formantes corresponden a http://ubumexico.centro.org.mx/sound/decampos_haroldo/galaxias/De-CamposHaraldo_Galaxias_01-e-comeco-aqui.mp3 y http://ubumexico.centro.org.mx/sound/decampos_ haroldo/galaxias/De-Campos-Haraldo_Galaxias_16-fecho-encerro.mp3, respectivamente.
} 
del libro. El tono más o menos parejo de la voz, muy segura, contrasta con el carácter improvisatorio del sitar, que no crea una melodía ni un ritmo fijos, sino más bien una ambientación. A pesar del carácter continuo de la prosa escrita sin puntuaciones, en la versión oral sí se marcan varias pausas. Esta segmentación contribuye a un mayor ordenamiento del flujo discursivo que visualmente parece más confuso.

Más adelante, el tono plácido del inicio (basado en la reiteración de las vocales "e" y "o" y las consonantes "m", "c" y "ç") se alterará al pasar la mitad del texto, en un punto más dramático producido por la secuencia de erres que se detiene súbitamente en "nulla res", dejando colgado al auditor antes de proseguir:

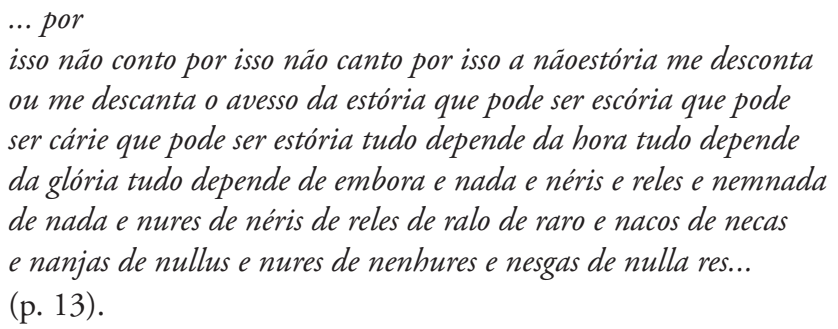

En el formante final, por otra parte, se observa a primera vista un carácter más activo y turbulento en las palabras, pues el despliegue de sus sonidos no es tan concentrado como en el inicial, sino más diverso, en parte por la mayor inclusión de vocablos extranjeros. La interpretación de Haroldo y su acompañamiento le otorga, sin embargo, un tinte más melancólico, como si quisieran remarcar el carácter conclusivo de la sesión. Al igual que en el caso anterior, la prosa pierde su condición de bloque compacto para adquirir un relieve, una expresividad que se vuelve más definida gracias a las intencionalidades que va dictando la voz.

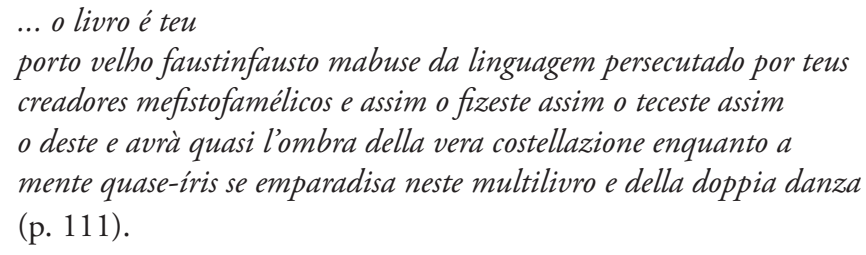

Confrontemos ahora estas lecturas con el primero de los términos hindúes aludidos por Haroldo: los raga. A diferencia de nuestro sistema tonal basado en las escalas (mayores y menores), los raga son modos melódicos que pueden entenderse como un color o afecto a partir de los cuales se desarrolla la improvisación, sin un tiempo marcado como el de un metrónomo, sino mucho más fluido. Esta música, 
como señala José Miguel Wisnik, es capaz de exaltar y llevar al éxtasis, la meditación o la danza (pp. 91-92). A pesar de que en un proyecto literario de esta envergadura y planificación es difícil pensar en un componente de improvisación (y menos de escritura automática, si recordamos el profundo rechazo de los poetas concretos al surrealismo), sí podemos considerar que esta estructura modal es comparable a la combinación de azar y control con que Haroldo proyectó la estructura de Galáxias. De hecho, en dos artículos publicados justamente en noviembre de 1963, Haroldo mencionaba algunos ejemplos de aleatoreidad en música, como los de Stockhausen y Cage, y citaba una obra de Pierre Boulez en la que el intérprete debe combinar los distintos formantes de la pieza, de la que el propio compositor francés señalaba "Essa integração do acaso seria feita sob a vigilância da inteligência criadora, para náo ceder lugar ao puro automatismo e ao caos" ( $A$ arte no horizonte do provável, p. 20$)^{6}$.

Esa combinación entre orden y azar se acentúa al escuchar la lectura del primer formante. Por una parte, tanto los acordes de apertura como el tono tan rotundo y afirmativo de la voz podrían remitirnos a la figura de un bardo que dará inicio a un poema épico cuyo desarrollo ya sabe de memoria. Por otra, si prestamos atención al contenido textual recordaremos que el ímpetu es distinto: más bien se trata de un comienzo que no acaba de comenzar, una especie de "tanteo", como cuando se muestran las cartas antes de barajarlas y repartirlas. Aquí sí podemos encontrar una relación con los raga: según refiere Inayat Khan, en la música hindú lo primero que se escucha es la afinación del instrumento, que es el modo en que el intérprete afina su alma y entra en contacto con la audiencia (p. 63). Es lo que ocurría también en la música instrumental del Renacimiento, con los ricercare o "tastar de corde", en los que precisamente se buscaba poner a punto el instrumento antes de comenzar a tocar "en serio". En ese sentido, el inicio que se demora en alcanzar su clímax, pareciera querer predisponernos de mejor modo para las epifanías que buscaba crear Haroldo en este libro de "ensayos".

En cuanto al mantra, nos obliga a entrar en un territorio más complejo pero fascinante. En su interesantísimo libro Sonic Theology. Hinduism and Sacred Sound, Guy L. Beck explica con mucho detalle las distintas maneras en que el sonido juega un rol capital en el hinduismo. Tanto él como J. Gonda en su artículo "The Indian Mantra" especifican y precisan los distintos sentidos de este y otros términos relacionados. Para efectos de esta pequeña investigación, me interesa solamente destacar que se trata de palabras o sílabas cuyo sonido es espiritual, que pueden ser consideradas como actos rituales en sí mismos, y que poseen un sistema distinto al del lenguaje cotidiano. Más importante aún son las consideraciones sobre su

\footnotetext{
${ }^{6}$ En la misma línea se puede citar la opinión de Rodolfo Mata, quien vincula Galáxias a la Teoría del Caos, y seńala que aquí "se da una mezcla armoniosa de azar y determinismo" (p. 149).
} 
sentido. Como indica Frits Staal: "The ritual meaning or function of such mantras does not lie in their language or even in their poetic or metrical structure, but in the sound with their themes and variations, repetitions, inversions, interpolations, and the particular distribution of their elements" (en Beck, p. 31).

Esta lógica de un sentido construido a partir del contexto, coincide con la pretensión de su autor, quien creía que las zonas oscuras y las palabras en otras lenguas podían cobrar un sentido a partir de su interrelación (aunque no podemos olvidar que los mismos comentarios a los poemas del CD, así como su constante afán sobreexplicativo, no confirman, sino más bien amenazan esta intención). Ese efecto parece lograrse en la versión oral, pues los distintos idiomas tienden a unificarse en la pronunciación del poeta, y la atención del auditor se concentra mayormente en las variaciones del flujo sonoro más que en cada palabra aisladamente. Estamos lejos, sin embargo, del tono plácido que generalmente asociamos a los mantras, especialmente en el contexto del yoga. De acuerdo al Hatha Yoga Prapidika, la señal del progreso en el yoga es que la voz se torna clara, melodiosa y resonante (Gannon, p. 134). La voz de Haroldo, por el contrario, suena potente, tensa, casi volcánica, en estas grabaciones.

Hay también una diferencia más importante, que es el tipo de repetición. En Galáxias asistimos, más allá de las reiteraciones de palabras y aliteraciones, a un discurso bastante variado y construido a partir de la suma, mientras que el mantra está constituido usualmente por una sola palabra repetida interminablemente, cuyo significado se pretende disolver en pos de otro sentido. Según explica Govinda Gopal Mukhopadhayaya "the main purpose of japa-continued repetition or muttering, whatever you may call it-is to kindle or arouse the fire in the Tantras is called kundalini. And, as these hard or concretized letters begin to melt, the aspirant or sadhaka begins to hear the Nada [sonido místico]. The words melt, leaving only a continuous flow of sound in the consciousness or experience of the aspirant" (en Beck, p. 132).

Suena divertida, en el marco de esta comparación, la imagen de estas letras "concretas" que se derriten, pero la verdad es que me parece difícil imaginarme que ello ocurra en estas Galáxias. La versión de Haroldo de Campos y Alberto Marsicano no provoca un vaciamiento de las palabras, sino más bien una saturación de su carga semántica y una superposición insistente de sus ecos en el auditor. Por eso, luego de avanzar un poco más en la posible relación de esta obra con los raga y los mantra, solo puedo concluir que esa comparación funciona de manera parcial, y desvinculada de toda carga espiritual. Estos referentes culturales mencionados por Haroldo no resultan, entonces, particularmente útiles para una mejor comprensión, sino que más bien responden a ese afán excesivamente abarcador y controlador con que muchas veces se refirió a su propia creación. De más está decir que esto no le quita un ápice a la fascinación envolvente y el placer puramente estético que produce la lectura de Galáxias, pero sí creo que puede aportar a la precisión de nuestros acercamientos críticos. En ese sentido, creo que 
este ejercicio podría sumarse a una evaluación más compleja acerca del modo en que su asimilación antropofágica de las literaturas orientales (particularmente la china y japonesa, como ya mencioné) no implica necesariamente la reformulación de las nociones occidentales de espiritualidad y subjetividad. En definitiva, estos textos y sus oralizaciones no constituyen una invitación mística ni menos la anulación de la conciencia, sino la inmersión en un viaje vitalista y libresco. A pesar de la constante combinatoria, mezcla y flexibilización a las que son sometidas, estas palabras se mantienen íntegras para entregar su mensaje en este mundo, no en otro.

\section{REFERENCIAS}

Aguilar, Gonzalo. Poesia concreta brasileña: Las vanguardias en la encrucijada modernista. Rosario: Beatriz Viterbo Editora, 2003.

Appratto, Roberto. "Una galaxia de signos", 1998. Disponible en: http://www. henciclopedia.org.uy/autores/Appratto/Haroldo.htm.

Beck, Guy L. Sonic Theology. Hinduism and Sacred Sound. Columbia, South Carolina, University of South California, 1993.

Block de Behar, Lisa (ed.), Haroldo de Campos. Don de poesía. Ensayos críticos sobre su obra. Montevideo: Librería Linardi y Riso, 2009.

Campos, Haroldo de. Xadrez de Estrelas. São Paulo: Editora Perspectiva, 1976.

Campos, Haroldo de. A arte no horizonte do provável. São Paulo: Editora Perspectiva, 1977 a.

Campos, Haroldo de. "Livro de Ensaios: Galáxias", Revista Iberoamericana, XLIII (98-99), enero-junio (1977b): 39-49.

Campos, Haroldo de. "La poesía Concreta según Haroldo de Campos" (entrevista), Vuelta, 25, (diciembre, 1978): 16-22

Campos, Haroldo de. Signantia: quasi coelum. São Paulo: Editora Perspectiva, 1979.

Campos, Haroldo de. "De la poesía concreta a Galaxias y Finismundo. 40 años de actividad poética en Brasil". Traducción de Carmen Salas y Rodolfo Mata, Vuelta 177, (agosto, 1991): 19-27. 
Campos, Haroldo de. "Occidente / Oriente. Uma conversa com Haroldo de Campos". Entrevista de Maria Esther Maciel. En Alberto Brandão Santos y Maria Antonieta Pereira (eds.), Trocais culturais. Belo Horizonte, Poslit/ UFMG,1999], en Zunái, http://www.revistazunai.com/entrevistas/haroldo_ de_campos.htm.

Campos, Haroldo de. De la razón antropofágica y otros ensayos. Selección, traducción y prólogo de Rodolfo Mata. México DF.: Siglo XXI Editores, 2000.

Campos, Haroldo de. "Haroldo de Campos: La pasión innovativa”. Entrevista de Julio Ortega. Taller de la escritura (conversaciones, encuentros, entrevistas). México D.F.: Siglo XXI Editores, 2000.

Campos, Haroldo de Galáxias. 2a edição. (incluye el CD Isto não é um livro de viagem), São Paulo: Editora 34, 2004.

Campos, Haroldo de. Crisantempo. Sáo Paulo: Editora Perspectiva, 2004.

Campos, Haroldo de. Metalinguagem \& outras metas. 4a edição. Sáo Paulo: Editora Perspectiva, 2006.

Campos, Haroldo de. O segundo arco-íris branco. São Paulo, Iluminuras, 2010.

Campos, Haroldo de. Galaxias. Traducción de Reynaldo Jiménez. Prólogo de Roberto Echavarren. Montevideo: La Flauta Mágica, 2010.

Costa Lima, Luz. "Arabescos de um arabista: Galáxias de Haroldo de Campos", $A$ aguarrás do tempo, Rio de Janeiro, Rocco, 1989.

Coward, Harold y Goa, David J. Mantra. Hearing the Divine in India and America. 2a edition. New York: Columbia University Press, 2004.

Cussen, Felipe. "Mantras y poesía sonora". Sala de lectura, 10 de enero de 2013. Disponible en: http://letraspuc.cl/index.php?option=com_content\&view= article\&id=1269: mantras-y-poesia sonora\&catid=81:publicaciones\&Item id $=458$.

Dick, André (ed.), Signâncias: Reflexóes sobre Haroldo de Campos. São Paulo: Risco Editorial, 2010. 
Echavarren, Roberto, Kozer, José y Sefamí, Jacobo (eds.), Muestra de poesía latinoamericana. México D.F.: Fondo de Cultura Económica, 1996.

Elialde, Mircea. Yoga. Inmortalidad y libertad. Traducción de Susana Aldecoa. Buenos Aires: Editorial La Pléyade, 1988.

Elialde, Mircea. Técnicas del Yoga. Traducción de Alicia Sánchez. Barcelona: Editorial Kairós, 1999.

Gannon, Sharon. "Yoga and Music". Arcana V. Music, Magic and Mysticim. In John Zorn (ed.), New York: Hips Road, 2010.

Gonda, Jan. “The Indian Mantra”, Oriens, 16, (december, 1963): 244-297.

Inayat Khan, Hazrat. The Mysticism of Sound and Music. Boston \& London: Shambhala, 1996.

Jackson, David K. (ed.), Haroldo de Campos. A Dialogue with the Brazilian Concrete Poet. Oxford, Centre for Brazilian Studies: University of Oxford, 2005.

Leminski, Paulo. "Prosa estelar", Anseios crípticos 2 (Curitiba, Criar Ediçóes), versión digitalizada por Digital Source, 2001, archivo PDF.

Mata, Rodolfo. "Barroco, caos y teoría de la información en la obra de Haroldo de Campos", Cuadernos Americanos 57, (mayo-junio, 1996): 141-49.

Milán, Eduardo. "Escritura, poesía, luz negra", Una cierta mirada. México D.F.: Juan Pablos Editor, 1989.

Milán, Eduardo. "El Odiseo brasileño. La poesía de Haroldo de Campos", Justificación material. México D.F.: Universidad de la Ciudad de México, 2004.

Motta, Leda Tenório da (ed.), Céu acima. Para um 'tombeau' de Haroldo de Campos. São Paulo: Editora Perspectiva, 2005.

Pérez, Fernando. The eye and the ear: Ezra Pound, Brazilian concrete poetry and their paideuma. Ph. D. Dissertation. New York: New York University, 2009.

Perloff, Marjorie. Unoriginal Genius. Chicago: The University of Chicago Press, 2010. 
Perlongher, Néstor. "El portuñol en la poesía", En Adrián Cangi y Reynaldo Jiménez. Papeles insumisos. Buenos Aires/Santiago: Arcos Editor, 2004.

Prazeres, Armando S. "Galáxia albina e Galáxia dark: uma inscriçấo poética de palavra no cenário eletrônico", Olhar, $1 / 8$ (2003): 36-39.

Santos, Lidia. Kitsch tropical. Madrid: Iberoamericana / Vervuert, 2001.

Sefamí, Jacobo. El destierro apacible y otros ensayos. México D.F.: Premià Editora, 1987.

Upanishads. Traducción de Juan Mascaró y José Manuel Abeleira. Barcelona: Debolsillo, 2010.

Verbovisual. A poesia concreta em música, CD, direção musical Cid Campos, Academia de Cultura / Base7 Projetos Culturais, 2007.

Wisnik, José Miguel. O som e o sentido. São Paulo: Companhia das Letras, 1999. 